\title{
Lacrimal Gland Carcinoma pT4c TNM Finding v8
}

National Cancer Institute

\section{Source}

National Cancer Institute. Lacrimal Gland Carcinoma pT 4C TNM Finding v8. NCI

Thesaurus. Code C140801.

Lacrimal gland carcinoma with involvement of adjacent structures, including sinuses,

temporal fossa, pterygoid fossa, superior orbital fissure, cavernous sinus, or brain and measuring more than $4 \mathrm{~cm}$ in greatest dimension. (from AJCC 8th Ed.) 